is the more astonishing that these little crustacea are found in almost every Bromeliad.

It cannot but sometimes happen that a few specimens must be washed away into other waters, as on the contrary, one may sometimes meet with a stray entrapped Cyclops that has slipped into the Bromeliads. Yet Fritz Müller has hitherto searched in vain for Elpidium in running waters, which producc, among other specics, Cypris, Cyclops, Canthocamptus, Chydorus, Alona, Camptocercus, Pasithca, Moina, Ceriodaphnia, Simocephalus, \&c. It seems not to flourish outside the Bromeliads.

[Translated from Kosmos, February, 1880. Elpidium comes somewhat near to Elpidia, Theel.-.-E. P. W.]

\section{ON THE PHYSICAL ASPECTS OF THE VORTEX-ATOM THEORY}

$\mathrm{I}^{\mathrm{N}}$

$\mathrm{N}$ all attempts to arrive at a satisfactory conception of 1 the ultimate constitution of matter, the grand difficulty of the atom with its capacity for executing vibrations, demonstrated by the spectroscope. The ancients, by assuming the atom to be infinitily hard, attempted in this way to get over the difficulty of indestructibility (or indivisibility), but thereby debarred all means of conceiving the "elasticity" of atoms, or their known powers of taking up vibrations of different periods.

When we consider the immense difficulty that there must have been in conceiving how an atom could be clastic (i.c., how its parts could be capable of free motion) and yet its paris be incapable of separation from each other, we may well excuse the attempt to explain indestructibility by the assumption of the quality of infinite hardness, unsatisfactory though it might be.

It is evident that if we are to renounce all idea of occult qualities of "clasticity," hardness, indivisibility, \&c., and purpose to explain the facts without recourse to postulates, we must assume the material substance of which our atoms are to be formed, to be itself entirely without any positive qualities, i.e., to be without elasticity, hardness, rigidity, \&c., and therefore to be frecly penetrable in all parts, or perfectly passive and inert. This is the perfect liquid of the rortex-atom theory. There may be some who would say that it is difficult to conceive of such a liquid. On the contrary, we venture to be able to prove that such a liquid alway's is conccived of whenever a liquid is thought of. Thus, does any one in conceiving of a liquid (water, for instance), regard the liquid as consisting of solid (i.t., more or less rigid) portions of matter sliding over each other [as we might conceive solid masses sliding past or through each other on a magnified scale]; and yet this is truly what the liquid (composed of molecules) is in the actual fact. In short it is not a "liquid" at all. Yet we conceive of it as liquid, i.e., frcely penetrable in all parts. We therefore contend that a perfect liquid (or true liquid) is what is alwery's conseived of, and therefore that there can be no difficulty in regard to the conception of the true liquid that forms the basis of the vortex-atom theory.

In the next place, it is an obvious condition to any consistent conception of matter that matter must possess extinsion, ${ }^{1}$ or occupy space, i.e., so that two portions of our liquid cannot occupy the same space at the same time. If, therefore, the liquid fills all space, it must be incompressible. This is, therefore, not an arbitrary postulate.

The next question naturally suggesting itself would be, how are portions of such a liquid to attain the properties that we recornise in atoms? We venture to think it will be conceded as evident that the only conceivable way (if it be admitted that the result is attainable at all) is through motion [for this is the only conceivable way in which the liquid can be affected]. The further inquiry would there-

${ }^{2}$ The quality of extension may even be regarded as included in the defini. tion: of matter. fore be, what would be the character of this motion? Now, in order to fulfil the condition that the atom itself can be brought to rest without losing its properties as an atom, it is evident that the motion of the material forming it must take place in such a way that the atom can remain in one spot, or be to our senses at rest, i.e., the material of the atom, although in motion, must not deviate from one spot. We ask if there is any other conceivable form of motion than rotary motion that would fulfil this condition? Hence the necessity for looking to rotary motion as the basis of the properties of the atom. In the next place a portion of material in rotation must rotate about an axis. If the ends of this axis were exposed, we should have two points at rest, which would forfeit the condition of motion being the essential basis of the external qualities of our atom. The question is, therefore, how is a portion of material to be in rotation about an axis, and yet not expose the ends of the axis? The only conceivable answer (as we think will be admitted) is that the rotating portion of material must have the form of a closed ring, or complete circuit, so that the axis has no ends. We therefore think it may be said beforehand that conceding that the problem of the atom can be solved at all (or if it be conceded that a fact can exist solely in virtue of the explanation that underlies it) then the problem could only conceivably be solved under the fundamental conditions above developed, i.c., under the condition of a portion of material (having no positive properties in itself) rotating in the form of a closed cirulit.

This (as is well known) is what has bcen found to satisfy the conditions for the atom by the application of mathematical analysis (without, apparently, that object having been in view at all), and in a manner the most remarkable in its completeness. It appears possible, in view of the above considerations, that a profound and competent thinker who had devoted himself to the subject might have arrived, even before the mathematical analysis had been applied, at the sole conceivable physical conditions that in principle could satisfy the problem of the atom (admitting the existence of the solution); but the mathematical analysis can of course alone make the fact of the solution apparent to us. It is related in the article on "The Atomic Theory of Iucretius" (North Britis/ Reviez, March, 1868) that Hobbes had arrived at the fundamental idea that the rotation of a portion of material must be the basis to the solution of the problem of the "elasticity" of the atom, without having applied any mathematics.

The difficulty of the mathematical side of the vortexatom theory is curiously contrasted with the simplicity of the physical side of the theory. If we suppose a cylindrical bar of india-rubber to be rotated about its longitudinal axis, and the bar (still rotating) to be bent round into a ring shape and the ends joined (the rotation of the material of the ring being alivays continued), then this may serve to illustrate in a simple way the motion of the material forming the vortex-atom. It is here apparent that the material of the india-rubber ring (in our illustrative case) may be in rapid motion while the ring itself preserves a fixed position in spacc. It would seem to be a pity if a spurious mystery should be allowed to envelope this subject, which is unwerthy of it, in view of the simplicity of its physical basis. No one doubts the difficulties that had to be surmounted on the mathematical side of the theory, but there is all the more reason on that account that the extreme simplicity of the physical side of the theory should be duly appreciated, and unnecessary obstacles not be thrown in the way of its adoption. The tendency to invest physical subjects with a halo of the occult [possibly partly attributable to the unfortunate introduction into physical science of the spiritualistic conception of "force"-in the sense of an action across space without the intervention of matter] has probably done more to hinder progress than any real difficulties. 
We shall simply state the facts of the mathematical analysis here, our business being more particularly with the physical side of the theory. First it is shown by incontrovertible mathematical proof that a portion of material having the motion above described possesses all the qualities of a solid. It is at the same time "elastic," or capable of changes of form when acted on through impact by other atoms-always tending to return to its symmetrical form when removed from constraint. It is, moreover, proved to be competent to execute vibrations of definite periods which it is the function of the spectroscope to measure. The atom thus constituted is demonstrated to be incapable of being divided or severed by the collisions of other similar atoms against it, and since this is the sole means of acting upon it, the long-standing riddle of indestructibility is thus simply solved, without the necessity for any postulate of infinite hardness. As the degree of hardness merely depends on the velocity of rotation of the material, it follows that the vortex-atom may possess any degree of hardness. Indeed, if we imagine the atom to be magnified up to visible scale, it might be conceived to be harder or more rigid than a ring of steel of the same dimensions, since the hardness of steel is limited by the resistance of the component atoms to displacement.

The centrifugal tendency of the rotating material of the vortex-atom is controlled by the exterior incompressible liquid, and as there is no friction [there being no ultimate solid parts in the rotating liquid to "catch" against the inclosing fluid walls], the rotating portion therefore glides smoothly over the incompressible liquid that surrounds it like a pipe. Indeed, if we leave out of our conceptions the portion of rotating liquid, then the surrounding liquid actually forms a complete pipe in the form of a closed ring. If the liquid in the pipe were to fly out, a temporary void would be formed in it, which is impossible in a liquid that already occupies all space. An idea of the resistance of such a rotating portion of material to bending may be got by attempting to deflect a gyroscope or spinning-top.

In the old idea of infinitely hard atoms there were difficulties in forming a satisfactory conception of what took place at the collision of two such atoms or how the rebound could effect itself (consistently with the conservation of energy). The following difficulty may also be mentioned :- Since two such atoms are supposed to be absolutely hard or unyielding, the area of contact at the collision would necessarily be merely a mathematical point. Now the intensity of a given pressure on a surface is inversely as its area; and accordingly, since the area is here a mathematical point (or infinitely small), the pressure attendant on the collision of the two atoms would require to be infinitely great. It may be a fair question how even an infinitely hard atom is to withstand the disintegrating influence of an infinite pressure. ${ }^{1}$

In the case of the vortex-atoms they yield somewhat at collision (without change of volume, of course), whereby the encounter takes place over a surface (not a point); and they rebound in virtue of their elasticity, due to the motion of the material forming them. ${ }^{2}$

There would seem to be a view to a certain extent prevalent that the vortex-atom theory essentially alters the basis of the old-established ideas of solid indestructible atoms surrounded by space in which they can freely move, to which so many have accustomed their conceptions, and worked upon to the successful discovery of new facts, and which ideas, therefore, they might be reluctant to abandon. This step, however, is not required at all. The main purpose of the vortex-atom theory is to explain the "elasticity"

I The fact of two such infinitely bard atoms being stopped in an infinitely short space at collision [for there is by hypothesis no gradual yielding] would by itself entail an infinite pressure in addition to the infinite pressure due to touching at a mathematical point.

2 The round of vor illustrated (as is known by the rebound of two smoke-rings from each other, or by the rebound of vortex rings in an ordinary (imperfect) licuid. of atoms, retaining substantially everything else appertaining to the old atomic theories, merely removing the unsatisfactory postulate of infinite hardness. For since the perfect liquid (outside the portions of it that form the atoms) opposes no resistance whatever to the passage of the atoms through it, or it is impossible to act on the exterior liquid, it is therefore in this respect as if a void existed outside the atoms. It is desirable, however, to note that the vortex-atom theory involves essentially the existence of the liquid outside the atoms, which performs important functions, but since this exterior liquid is proved to be incapable of appealing to our senses in any way, it therefore in that respect may be said to play the part of a void, The exterior liquid of the vortex-atom theory corresponds to the roid space of the theory of Lucretius. With the above qualification, therefore, it may be allowable, when we are not specially dealing with the problem of the constitution of the atom itself, to leave out of our conceptions the presence of the exterior liquid: that which we call "matter" being the atoms, and not the exterior liquid. In all practical problems of physics therefore (apart from the problem of the constitution of the atom), we may properly regard the atoms simply as elastic indestructible solids moving freely in space. Moreover, since the motion of rotation of the material of the atom is incapable of transference, and cannot appeal to our senses, and this motion does not in any way alter the position of the atom in space [but it is exactly as if the atom itself were at rest]; we can therefore, if we like, leave this rotatory motion out of our conceptions, merely keeping in view the result produced by the rotation, viz., the sharply-defined elastic indestructible solid thereby formed. The function of the modern theory is accordingly not to destroy the atomic theory of the ancients, but rather to support it, by explaining how such indestructible bodies can exist, without recourse to the unacceptable postulate of infinite hardness. This old theory of the atomic constitution of matter was really too firmly grounded on reason and observation, as that one should suppose that its very foundations could be shaken.

Broadly and generally, therefore, in practical problems of physics, the essential points to recognise are that atomsor molecules -are elastic indestructible bodies, capable of rebounding from each other without loss of energy, and of executing vibrations of fixed periods. The existence of this elasticity is a fact so definitely proved by the spectroscope, which actually measures the number of vibrations executed per second by molecules, that it would become a question to explain this fact, even if the vortexatom theory had not been proved to be capable of affording a complete explanation of it. Indeed, not only is the theory capable of doing this, but the vibrating capacity possessed by molecules is shown to be a necessary consequence of the theory, so that, therefore, the fact might even have been deduced à priori. Considering how enormously difficult it appeared to account for this fact at one time, or how impossible it seemed to reconcile the mobility of the parts of a molecule with the inseparability of these parts by the most energetic collisions, and how an explanation of this fact was at one time sought after, it would appear not too much to expect that those who hesitate to accept the explanation given by the vortex-atom theory, should endeavour to define for themselves wherein their grounds of objection lie. For if the explanation of a fact be admitted to be substantially complete, it would be at least unreasonable to look for more. The question might also suggest itself as a fitting one to any impartial inquirer, whether any other solution to the problem of the constitution of the atom is in principle conceivable, or whether [as in the case of many other physical problems, the constitution of the ether, for instance] but one solution is conceivable (or we have no choice at all). It cannot be said at least that the theory of vortex-atoms, or its physical side, is not simple, dealing 
as it does with the mere rotation of a portion of matter. ${ }^{\mathrm{I}}$ It is so far recognised that simplicity of the means to the end is a general characteristic of nature. No doubt there may be difficulties in the mathematical development of the subject; but if an atom be once proved to be elastic and indestructible, that fact surely goes very far to supply all we want for the practical applications of the theory. Of course there may be some refinements that may present great mathematical difficulties. For instance, Prof. Tait in his work, "Lectures on some Recent Advances in Physical Science," mentions a case where a vortex-ring is supposed to come into collision with another in such a way that the motion is not symmetrical in relation to the axis, and it is cited as an almost insurmountable difficulty to find what exactly takes place (in regard to particular vibrations or rotations developed, possibly). But one might ask, is it necessary to know this for practical problems of physics? We may know broadly that vibration or rotation is developed, and if so (apart from the abstract interest of the question), do we want to know precise quantitative details for practical purposes? It might for example be extremely difficult to determine mathematically the exact deformation or changes of form (vibrations, \&c.) that a steel ring underwent when thrown against the hard surface of an anvil; but the practical question is, do we want to be acquainted with this for any ordinary problem that might occur, or in order to appreciate the general principles of impact, for instance? So in the case of vortex-atoms, no doubt many instances might be cited when it would be difficult to ascertain precise results, but the practical question is, Does this prevent our applying the theory to ordinary physical problems, ${ }^{2}$ or to dynamical phenomena involving questions of principle? For possibly it may not be necessary to know the exact vibrations developed at a collision (for instance), provided we recognise the fundamental point that energy is conserved, and that the atoms can rebnund from each other like perfectly elastic solids. It would be a pity if the mere difficulty of arriving at precise mathematical results of a refined character, should be mistaken by some for mystery, or it would be a thing to be regretted if there should be any tendency to throw a veil of the "occult" over what in its physical basis (at least) is very simple, this procedure only hindering progress and rendering a closed book what night be a most interesting branch of mechanics.

The investigations regarding the perfect liquid bave already (as is known) thrown some important light on the important practical question of the resistance of ships. Mr. Froude has especially devoted himself to these inquiries. The old idea that a ship (or more correctly a

${ }^{I}$ It would seem to be thought by some that the primary ring form of the vortex-atom involves something complicated in it. I venture to think that this is only one of those first impressions, which will disappear on reflecting on the subject. First, many facts strongly indicate that matter possesses a more or less open structure (or is highly porous). These ring molecules would give matter an cpen structure. It would seem also independently probable that a molecule should have no more material in it than is essential to give it a certain amcunt of extension, or to make it occupy a certain range of space. Why should we suppose that waste or apparent superfluity of material in a molecule that a solid structure throughout would involve Dues not this violate one of the fundamental principles of large scale architecture, where superfluity of material is recognised as one of the worst faults, and mechanical principles are admittedly independent of scale? The ring shape for the atom is evidently the simplest elementary form to satisfy the condition for the maximum of extension combined with the minimum waste or expenditure of material. In view of these considerations, the ring-shape, the primary form required by the vortex-atom theory, may seem in itself independently probable. Indeed, it seems a remarkable fact that the main conditions inevitably led up to by this theory by a rigid mathematical process, are precisely those that independent observations support, $(\mathrm{I})$ the indestructibility of the atom, illustrated by chemistry and numerous facts, $(2)$ the elasticity of the atom, proved by the spectroscope, $(3)$ the open structure of the atom in harmony with the transparency of some bcdies to light, the free passage of the magnetic disturbance through all bodies, and numerous other facts-not to mention the phy:ical theory of gravity. In short, it would appear tnat it would be necessary to infer the existence of indestructible e:astic atoms of open structure, even if the vortex atom theory (which explains this fact) ad not been invented.

2 The writer himself has seen from German comments on Prof. Tait's work, that the passage above referred to [German translation] has been mentioned were of such a nature as to prevent the practical adoption of the theory. totally immersed body, such as a fish) encountered a mysterious resistance in addition to the mere friction of the molecules of water on its sides, is now known to have been a pure delusion. If it were not for the fact that the water consisted of molecules or ultimate rigid parts which are caught and put in motion by the rough sides of the ship, there would be demonstrably no resistance at all. Hence the absence of resistance in a true liquid (which is not formed of ultimate rigid parts or molecules). If the molecules or ultimate rigid parts of which an ordinary "liquid" consists, were to be liquefied, a being immersed in it would (if conscious) imagine be was surrounded by empty space.

The late.Prof. Clerk Maxwell in a review of the theory of vortex-atoms in the "Encyclopædia Britannica" for 1875, under the word "Atom," makes the following remark on the theory :-

"But the greatest recommendation of this theory from a philosophical point of view, is that its success in explaining phenomena does not depend on the ingenuity with which its contrivers 'save appearances' by introducing first one hypothetical force and then another. When the vortex-atom is once set in motion, all its properties are absolutely fixed and determined by the laws of motion of the primitive fluid, which are fully expressed in the fundamental equations. The disciple of Lucretius may cut and carve his solid atoms in the hope of getting them to combine into worlds; the follower of Boscovich may imagine new laws of force to meet the requirements of each new phenomenon; but he who dares to plant his feet in the path opened out by Helmholtz and Thomson has no such resources. His primitive fluid has no other properties than inertia, invariable density, and perfect mobility, and the method by which the motion of this fluid is to be traced is pure mathematical analysis. The difficulties of this method are enormous, but the glory of surmounting them would be unique" [p. 45].

Much misapprehension would seem to exist in regard to the physical side of the theory, especially in Germany, where the mathematical investigations out of which it sprung, had their origin. Some appear to be unable to conceive how motion should take place in a material substance continuously filling space, losing sight of the fact that the liquid outside the atoms plays the part of a void (in so far as it cannot appeal to our senses)-or it is only the atoms that affect our perceptions. Others fail totally to appreciate the simplicity of the physical side of the theory, and seem to think it involves arbitrary postulates, vihereas the main peculiarity of the theory is its freedom from positive assumptions, inasmuch as the theory evolves all the properties of matter out of the motion of a material substance, which without this motion has no positive qualities at all, and could not appeal to our senses. The fact seems to be overlooked that if we renounce the occult quality of rigidity in the atom, we have no other resource than a liquid (i.e., a substance without rigidity). Much of the misunderstanding on the subject may no doubt be due to the scarcity of the literature relating to it, and the extreme brevity and absence of detail or attempt to assist the conceptions regarding the physical side of the theory. This want the author himself has much felt, and having been at considerable trouble to render clear his own conceptions as far as he could, he has thought that the result of this analysis might not perhaps be unacceptable in the form of a paper on the physical aspects of the theory. ${ }^{2}$ For there are no doubt

I The writer has had personal experience cf this, partly through correspondence, and partly through the literature relating to the subject. Quotaappreciation of the physical points of the theory at their true value and significance.

'As regards sources of info mation as to the vortex-atom theory, the following may be mentioned. Sir William Thomson, "On Vortex-Atoms," Brit. $x 875$.' The theory is dealt with to some extent in a popular manner in 
many investigators in the paths of natural science who may find some difficulty in realising the physical basis and real bearings of the theory, and who nevertheless take a rational interest in the solution it is capable of affording to some of the greatest difficulties of molecular physics. The whole structure of physics may be said to rest upon a molevular basis, and therefore the importance of a right view of this basis cannot be over-estimated. The old theory of perfectly rigid molecules put an immense difficulty in the way of the development of physical results upon such a groundwork. A theory of elastic molecules therefore becomes of the utmost importance as a practical working hypothesis, and the accordance with observation of new results predicted from this hypothesis as a basis, will then form additional confirming illustrations of its truth. The removal of any misunderstandings that might be obstacles in the way of the use of the vortex-atom theory as a working hypothesis becomes, therefore, a point of considerable importance. Those more especially who have handled the spectroscope and viewed the exquisite precision of its results, become impressed with the certainty of the groundwork upon which their molecular studies are based, and no less imbued with the conviction of the existence of that explanation that forms the basis of the facts that are recorded with such unfailing accuracy.

\section{S. TOLVER PRESTON}

\section{COMPARATIVE ANATOMY OF MAN ${ }^{1}$}

$$
\text { I. }
$$

THE great scope and interest of the subject of anthropology, as weil as its most convenient subdivisions, are well illustrated by the prospectus of the teaching at the Anthropological Institute of Paris. There are at present six chairs :-(I) Comparative Anatomy in Relation to Anthropology, by Broca; (2) Biological Anthropology, or the Application of Anatomy and Physiology to Anthropology, by Topinard; (3) Ethnology, or the Study of the Races of Man, by Dally ; (4) Linguistic Anthropology, by Hovelacque ; (5) Palæontological and Prehistoric Anthropology, by Mortillet ; and (6) Demography, which includes what we commonly call social and vital statistics and Medical Anthropology, by Bertillon. These subjects are publicly taught in a school supplied with all necessary appliances, founded partly by private munificence, but also liberally subsidised by the Municipality of Paris and the Department of the Seine. There is also at Paris a complete course of general anthropology given yearly by $M$. de Quatrefages in connection with the magnificent museum at the Jardin des Plantes. To these institutions we have nothing comparable in England, and neither at our Universities or elsewhere is any branch of anthropological science systematically taught. The present lectures only embrace a small portion of one of the six subdivisions enumerated above, that of biological anthropology. This science is purely one of observation, and in proportion as the materials upon which our observations are founded are multiplied, so will the value of the observations be increased. These materials are collected in museums, which at present in this country are not so complete as might be desired. The largest public collection is that of the College of Surgeons, containing about 1,200 crania of different races; the largest private collection is that of Dr. Barnard Davis, of Shelton in Staffordshire, considerably exceeding that of the College both in number and variety of specimens. Happily these are about to be united, and, under the care of the Council of the College, will be made accessible to all who wish to pursue the study of anatomical anthropology. an article on "The Atomic Theory of Lucretius," North British Review,
March, r868, also by Prof. Tait, in his work "Lectures on Some Recent Advances in Physical Science." x Abstract Report of Prof. Flower's lectures at the Royal College of
Surgeons, March I to March I9, on the Comparative Anatomy of Man.
Besides the Barnard Davis collection, only a small portion of which has as yet been received, one of the most important additions to the museum since the last course of lectures is a series of skulls collected in the Fiji Islands in 1876 by Baron Anatole von Hügel, forming part of a donation made by Mr. Erasmus Wilson. They consist of sixteen crania of the Kai Colos, or mountaineers of the interior of the western portion of Viti Levu, and five crania from the eastern coast and small islands adjacent The inhabitants of the Fiji group are generally described by ethnologists as a mixed race, compounded of $\mathrm{Me}$ lanesians and of brown Polynesians, as the islands are situated on the confines of the territories inhabited mainly by these two races, and the few crania hitherto accessible have favoured this view. Those, however, of the Kai Colos brought home by Baron von Hügel, and which probably represent the most primitive native population of the islands, show all the characters of the purest Melanesian type, without the slightest trace of Polynesian mixture. Their purity is shown by their wonderful similarity, and by their very peculiar and stronglymarked characters, discernible with equal facility in both sexes and at all ages. They are large, the average capacity of eight adult males being $I, 482$ cubic centimetres; and with muscular ridges and impressions strongly developed. In proportion to their length, they are the narrowest crania known, having an average latitudinal index of only $66^{\circ}$. Not one has the index so high as $70 \circ$, and in one it descends as low as $6 \mathrm{I}^{\circ} 9$, which is below that of any other normal skull in the collection. The heigbt in all very considerably exceeds the breadth, the avcrage altitudinal index being $74^{\prime} \mathrm{I}$. They thus belong to the most strongly marked hypsistenocephalic type. The zygomatic arches are very wide compared with the cranium. The brow ridges are strongly marked, though less so than in the Australians. The orbits are low and quadrangular, the nasal bones short, though rather prominent, and the nasal aperture wide (index $57^{\circ} \mathrm{I}$ ), the jaws prognathous, though not to an extreme degree, and the teeth large. The skeleton of the face thus conforms with what is generally found in the Melanesians or Oceanic negroes, but the features are on a larger scale and more strongly pronounced than in the inhabitants ot many of the New Hebridean and Papuan islands. The skull of the Tongans and Samoans, living on islands scarcely 300 miles from the Fijis, presents the greatest possible contrast to that just described. It is short and round (latitudinal index $82^{\circ} 6$ ), the orbits are rcund, the nasal bones long and flat, and the aperture narrow (index $44^{\circ} 3$ ), and the jaws are not prognathous. It is well known that for a long time the Tongans have been in the habit of visiting the Fijis, especially the smaller islands to the east of the group, and that there is in the inhabitants of that region a considerable infusion of Tongan blood. Five skulls of natives of the small island of Vanua Balavu, where this influence is supposed to prevail, show a distinct deviation in every character from that of the Kai Colos, and these deviations are, without exception, in the direction of the Tongan or Polynesian type. The average latitudinal index is raised to $71^{\circ} 9$; the nasal index is $50^{\circ}$, the orbits intermediate in form, and the prognathism much reduced. No skulls have as yet been examined from the second large island, Vanua Levu, and the numbers of those just described are, perhaps, not sufficient to draw any great conclusions from, but, as far as they go, they tend to show that, so far from the Fijians generally being a mixed race, the mass of those that inhabit the interior of the large islands are remarkably pure, and of the Melanesian or Papuan type in its most characteristic, almost exaggerated, form, but that the natives of the coast districts and outlying islands to the east show certain tendencies towards the brown Polynesian type, and as these are the people with whom European visitors to the Fijis have mostly come 